\title{
Biobanques: il est urgent d'investir dans une loi fédérale
}

\author{
Dominique Sprumont ${ }^{\mathrm{a}}$, Charles Joye ${ }^{\mathrm{b}}$, Astrid Pilottin ${ }^{\mathrm{c}}$ \\ a Professeur en droit, directeur-adjoint de l'Institut de droit de la santé, Université de Neuchâtel, Vice-directeur de la Swiss School of Public Health et \\ de I'Ecole romande de santé publique \\ b Professeur remplaçant (UNIL/EPFL) - droit de la recherche technologique et propriété intellectuelle, membre de la Commission cantonale d'éthique \\ de la recherche sur l'être humain (VD), avocat \\ c Etudiante, Master en droit, orientation de droit de la santé et des biotechnologies et propriété intellectuelle, Université de Neuchâtel
}

1 Par biobanque, il faut entendre ici une collection organisée d'échantillons biologiques humains et de données associés.

2 Département fédéral de l'intérieur, Rapport concernant les résultats de la procédure de consultation portant sur l'avantprojet de disposition constitutionnelle et de loi fédérale relative à la recherche sur l'être humain, Berne, février 2007.
La Suisse a toujours été à la pointe de l'innovation, notamment dans le domaine de la santé. Pourtant, en ce qui concerne les biobanques ${ }^{1}$, elle risque de souffrir d'un retard important par rapport à ses voisins. Le printemps 2016 fut riche en rapports et textes publiés sur ce sujet. Une conclusion semble réunir un large consensus: une loi spécifique doit être évaluée et, le cas échéant, adoptée pour améliorer la réglementation des biobanques.

\section{Biobanken - ein entsprechendes Bundesgesetz ist dringend erforderlich}

Biobanken sind ein neues, vielversprechendes Instrument, um unser Verständnis vom menschlichen Körper und die Forschung im Bereich der öffentlichen Gesundheit voranzubringen. Trotz mehrerer diesbezüglicher Initiativen könnte unser Land im internationalen Vergleich ins Hintertreffen geraten. In der Tat zeigen mehrere Berichte und Empfehlungen, die 2016 sowohl in der Schweiz als auch im europäischen und im internationalen Ausland veröffentlicht wurden, dass der Bedarf zur Harmonisierung nationaler, europäischer und internationaler Netze stetig zunimmt. Trotz erheblicher Bemühungen der betroffenen Stellen bietet das Humanforschungsgesetz (HFG) keine Regelungen für Biobanken und es bestehen juristische Definitionslücken, vor allem was die Eigentumsrechte, den Datenschutz und die Kontrolle anbelangt. Die Annahme eines Bundesgesetzes über die Biobanken wäre möglicherweise eine einzigartige Chance, die diesbezüglich bestehende Unsicherheit zu reduzieren, indem die Respektierung der erforderlichen Kontrollvorschriften zum Schutze der Teilnehmenden garantiert und Qualitätsforschung im Interesse der Patienten und der öffentlichen Gesundheit gefördert würden.

\section{Pourquoi une législation ?}

Aujourd'hui, des biobanques publiques ou privées, commerciales ou de recherche sont implantées sur le territoire helvétique et leur nombre connaît une véritable explosion. Ce phénomène n'est pas spécifique à la Suisse et se déroule simultanément dans nombre de pays. Il existe un important intérêt économique et scientifique au développement des biobanques. L'ordre juridique se doit d'intervenir pour protéger les droits fondamentaux, mais également pour ne pas étouffer cet élan de recherche et permettre à la Suisse de garder sa place mondialement reconnue pour ses avancées médicales. Le développement des biobanques est lié à celui de la recherche, particulièrement dans le domaine de la santé personnalisée. Un retard dans l'encadrement de cet outil de recherche provoquerait immanquablement des déficits dans le développement de ce domaine. Mais pourquoi une législation paraît-elle nécessaire?

Le cadre légal actuel suisse des biobanques repose sur une série de lois qui couvre chacune de manière ponctuelle et partielle les grands enjeux que soulèvent les biobanques: Constitution fédérale [1], Code civil [2], Code pénal [3], loi sur la protection des données [4], pour citer les textes généraux, mais aussi loi sur la recherche sur l'être humain (LRH) [5], loi sur l'analyse génétique humaine (LAGH) [6], loi sur l'enregistrement des maladies oncologiques [7], loi sur le dossier électronique du patient [8], etc. En ce qui concerne la LRH, la procédure de consultation qui a précédé son adoption avait justement mis en lumière la nécessité de ne pas y régler de manière exhaustive les biobanques mais de se limiter aux questions de recherche avec des échantillons biologiques et des données personnelles ${ }^{2}$. Cette loi est ainsi composée de quelques d'articles à propos de la réutilisation de matériel biologique et de données, mais uniquement dans le cadre de projet de re- 


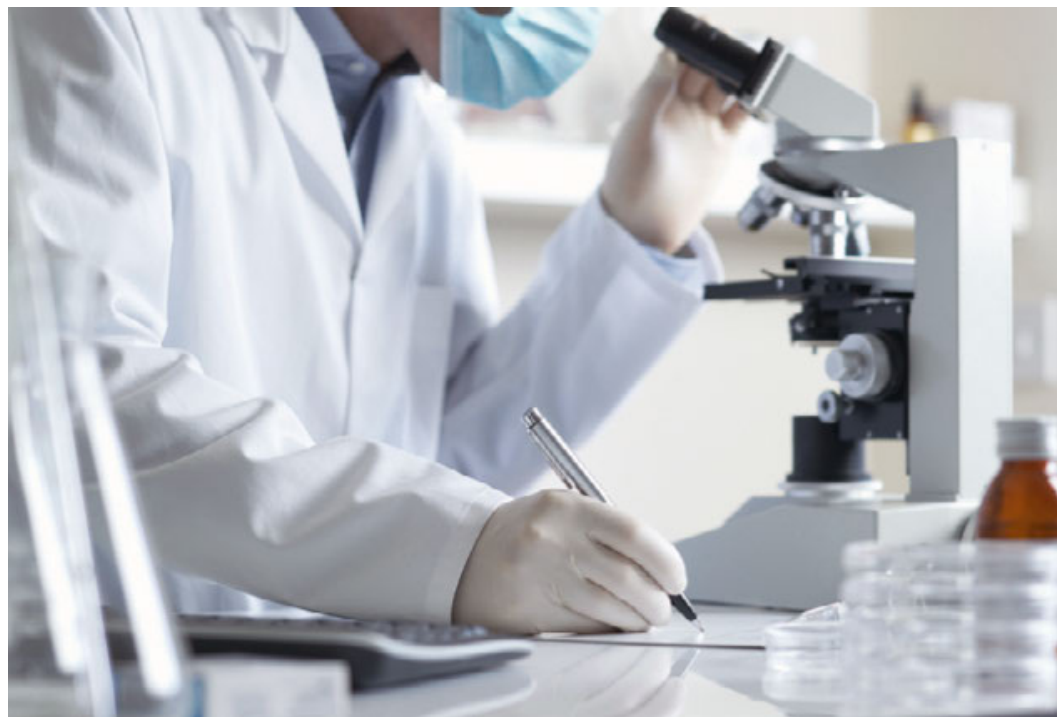

Il existe un important intérêt économique et scientifique au développement des biobanques.
Cette commission n'est pas le seul acteur suisse à pointer du doigt le flou de la LRH et à suggérer une intervention du législateur. Les milieux intéressés ont été les premiers à se préoccuper des carences de la LRH et à proposer des règles de bonne pratique afin d'y remédier partiellement. A ce titre, on peut saluer les initiatives de certains acteurs ou organismes intéressés, comme le cadre proposé par la Biobanque institutionnelle de Lausanne, ou encore celui édité par Swissethics sous le titre: Explication relative à l'utilisation ou la réutilisation de matériel biologique et de données liées à la santé pour la recherche biomédicale (juillet 2016). De même, dans son dernier Bulletin d'août 2016 [12], l'ASSM annonce le développement d'un formulaire de consentement qui pourrait être utilisé dans tous les hôpitaux participant à la constitution de biobanques. A l'échelon national, nous pouvons encore mentionner la Swiss Biobanking Platform (SBP) qui vise à coordonner différentes biobanques du pays avec un objectif d'harmonisation de leur fonctionnement [13], une organisation par ailleurs composée de membres des cinq hôpitaux universitaires suisses. Ce projet s'intègre du reste dans un réseau plus large, le Swiss Personalized Health Network qui partage des objectifs communs [14].

Ces initiatives et propositions d'autorégulation font écho à différentes positions adoptées en Europe, notamment grâce à l'impulsion de mise en réseau de biobanques au niveau européen comme la Biobanking and Biomolecular Resources Research Infrastructure (BBMRI) ou l'Eurobiobank, nécessitant une certaine harmonisation et un consensus afin de permettre une plus grande pérennité dans la circulation des données. A l'échelle européenne, on retrouve également la recommandation, $\operatorname{Rec}(2016) 6$, du 11 mai 2016 du Conseil de l'Europe. Celle-ci est une révision, beaucoup plus complète, de la recommandation (2006)4 sur la recherche utilisant du matériel biologique d'origine humaine. Elle prend en compte de nouveaux développements dans le domaine des biobanques et offre une vision claire des principes applicables y compris en matière de gouvernance.

\section{Une loi spécifique permettrait un déve- loppement harmonieux des biobanques dans le respect des participants}

Un consensus est d'ailleurs trouvé entre cette recommandation et l'Association médicale mondiale qui a adopté le 22 octobre 2016 à Taipei une déclaration sur les considérations éthiques concernant les bases de données sur la santé et les biobanques, dite la déclaration de Taipei [15] en complément de la déclaration 
d'Helsinki. Cette déclaration de portée universelle s'aligne sur la conception du consentement retenue par le Conseil de l'Europe et met aussi l'accent sur la nécessité d'adopter des règles claires en matière de gouvernance des biobanques. Ce projet a reçu l'aval de la Commission centrale d'éthique de l'ASSM. A son article 24, la déclaration de Taipei exhorte les autorités à adopter les politiques et les lois nécessaires afin de garantir et mettre en œuvre les principes qu'elle consacre.

Le débat sur la nécessité de l'adoption d'une loi spécifique dans nos pays voisins a déjà amené certains d'entre eux à adopter une règlementation. Ces pays ont su développer des biobanques de pointe. On retrouve celles-ci en Angleterre, en Belgique, en Islande ou encore en Espagne. Il semble donc qu'il ne s'agisse pas d'une complication mais bien d'un bénéfice pour les chercheurs. Un manque d'uniformité peut empêcher certaines coopérations entre les chercheurs, ce qui a un impact à long terme sur la compétitivité et la viabilité des projets de recherche [16]. Cette nécessité d'une gouvernance commune en Suisse serait un outil facilitant la mise en réseau des biobanques. Or, une loi spécifique permettrait de ne pas travailler de manière isolée tout en imposant un certain contrôle tout au long de l'exploitation des biobanques. Le débat sur une loi spécifique en Suisse devrait être lancé, et permettrait par la même occasion de vulgariser le sujet.

Il paraît ainsi urgent d'initier un processus d'évaluation visant à l'adoption d'une loi fédérale spécifique aux biobanques, en tenant compte des initiatives importantes prises par les milieux intéressés. La loi fédérale sur les biobanques devrait garantir la protection des participants, la santé publique, ainsi que la liberté scientifique et économique. Cette loi pourrait servir de fondement à la mise en réseau des biobanques existantes en Suisse et à la création d'une biobanque populationnelle nationale. Elle devrait fixer les exigences minimales applicables à toute biobanque, dans le secteur public comme dans le secteur privé. L'investisse- ment dans une telle loi fédérale fait partie des infrastructures essentielles dont la Suisse a besoin pour soutenir l'innovation tout en faisant face aux défis de santé publique, éthiques et juridiques que constituent la réforme de notre système de santé et le développement annoncé de la santé personnalisée.

\section{Crédit photo}

(c) 18percentgrey | Dreamstime.com

\section{Références}

1 Constitution fédérale de la Confédération suisse du 18 avril 1999 (RS 101).

2 Code civil suisse du 10 décembre 1907 (RS 210).

3 Code pénal suisse du 21 décembre 1937 (RS 311.0).

4 Loi sur la protection des données du 19 juin 1992 (RS 235.1).

5 Loi sur la recherche humaine du 30 septembre 2011 (RS 810.30).

6 Loi sur l'analyse génétique humaine du 8 octobre 2004 (RS 810.12).

7 Loi sur l'enregistrement des maladies oncologiques, entrée en vigueur prévue entre janvier 2018 et janvier 2019 (Mise à jour du 02.06.2016) http://www.bag.admin.ch/themen/gesundheitspolitik/10374/index.html?lang=fr (dernière consultation 22 septembre 2016).

8 Loi sur le dossier électronique du patient, entrée en vigueur prévue en 2017, adoptée par le Parlement le 19 juin 2015 (Mise à jour du 20.04.2016) http://www.bag.admin.ch/themen/gesundheitspolitik/10357/10360/index.html?lang=fr (dernière consultation: 22 septembre 2016).

9 ASSM, Directives médico-éthiques relatives aux biobanques : prélèvement, conservation et utilisation de matériel biologique humain du 23 mai 2006.

10 Commission nationale d'éthique pour la médecine humaine (CNE), Les biobanques destinées à la recherche - Prise de position n 24/2015, Berne, décembre 2015 http://www.nek-cne.ch/fileadmin/nek-cne-dateien/F_Broschure_ NEK_Biobank.pdf (dernière consultation : 22 septembre 2016)

11 CNE, N 135 et 136 .

12 ASSM, Consentement général: un modèle uniforme pour faciliter la recherche sur tout le territoire suisse, Bulletin 03/2016, pp. 1 ss.

13 http://www.swissbiobanking.ch (dernière consultation: 22 septembre 2016).

14 http://www.samw.ch/en/Projects/Personalized-Health.html (dernière consultation: 22 septembre 2016).

15 http://www.wma.net/en/30publications/10policies/d1/index.html (dernière consultation: 24 octobre 2016.

16 Kaye J. Do we need a uniform regulatory system for biobanks across Europe ? European Journal of Human Genetics (2006) 14, 245-48, 23 novembre 2005 http://www.nature.com/ejhg/journal/ v14/n2/full/5201530a.html; Caroline Trouet and Dominique Sprumont, Biobanks: Investing in Regulation. Baltic Yearbook of International Law 2 (2002), pp. 3-19. 